\title{
Numerical Solution for Elliptic Interface Problems Using Spectral Element Collocation Method
}

\author{
Peyman Hessari, ${ }^{1}$ Sang Dong Kim, ${ }^{2}$ and Byeong-Chun Shin ${ }^{3}$ \\ ${ }^{1}$ Department of Mathematical Sciences, Ulsan National Institute of Science and Technology, Ulsan 689-798, Republic of Korea \\ ${ }^{2}$ Department of Mathematics, Kyungpook National University, Daegu 702-701, Republic of Korea \\ ${ }^{3}$ Department of Mathematics, Chonnam National University, Gwangju 500-757, Republic of Korea
}

Correspondence should be addressed to Byeong-Chun Shin; bcshin@jnu.ac.kr

Received 24 February 2014; Revised 26 May 2014; Accepted 2 June 2014; Published 24 June 2014

Academic Editor: Sofiya Ostrovska

Copyright (C) 2014 Peyman Hessari et al. This is an open access article distributed under the Creative Commons Attribution License, which permits unrestricted use, distribution, and reproduction in any medium, provided the original work is properly cited.

\begin{abstract}
The aim of this paper is to solve an elliptic interface problem with a discontinuous coefficient and a singular source term by the spectral collocation method. First, we develop an algorithm for the elliptic interface problem defined in a rectangular domain with a line interface. By using the Gordon-Hall transformation, we generalize it to a domain with a curve boundary and a curve interface. The spectral element collocation method is then employed to complex geometries; that is, we decompose the domain into some nonoverlaping subdomains and the spectral collocation solution is sought in each subdomain. We give some numerical experiments to show efficiency of our algorithm and its spectral convergence.
\end{abstract}

\section{Introduction}

Second order elliptic interface problems have many applications in engineering and science, in particular in fluid dynamics and material science [1-5]. Multiphase flow, crystal growth, modeling of the Stefan problem of solidification process, composite materials, cell and bubble deformation, and fiber suspension are few examples of the interface problems to be mentioned. Owing to discontinuity of coefficients of differential equations and singular source term, the solution and its derivative of interface problem are nonsmooth or even discontinuous across the interface. Hence usual numerical methods for partial differential equations do not work efficiently for these problems. Such kind of problems has attracted attention of scientists from both theoretical and computational approach over the past decades. Several high-order methods have been developed to deal with interface problem in terms of discontinuous coefficients and/or singular source terms. In [6] LeVeque and Li have investigated second order accurate finite difference method for one-dimensional interface problem. For one-dimensional problem, the standard finite element method can be used and if the interfaces lie on the grid point, second order accurate solution can be gained [7]. However by modifying basis function, Li obtained a second order accurate finite element method in the maximum norm in [7]. The finite difference and finite element method for one-dimensional problem are not much different. In [8], Shin and Jung used spectral collocation method and radial basis function method for one-dimensional interface problems and obtained very accurate solutions using the similar ideas given by Loubenets et al. in [9]. For two-dimensional elliptic interface problem, in [6], the immersed interface method (IIM) is developed for an elliptic interface problem with discontinuous coefficients and singular source term. By adding an extra node to the standard finite difference 5-point stencil, Huang and Li derived a second order accurate method in [10]; but the coefficient matrix is nonsymmetric and the method is stable for problems with piecewise constant coefficient. This problem is solved by a new version of IIM in [11]. In [12], Angelova and Vulkov presented second and fourth order finite difference scheme for elliptic problem with intersecting interface which has discontinuous variable coefficient and singular source term. However they assumed that interfaces are straight lines. In [13], Li et al. investigated finite element method based on Cartesian triangulation for interface problem involving discontinuous coefficients which is second order accurate in the maximum norm. Hanke and Loubenets proposed 
an immersed finite element method for elliptic interface problem with discontinuous coefficients and singular source term and present its second order convergence in $L^{2}$ norm in [14]. Kwak et al. have introduced an immersed finite element method based on the "broken" $P_{1}$-nonconforming piecewise linear polynomials on interface triangular element having edge average as degrees of freedom in [15]. These linear polynomials are broken to match the homogeneous jump condition along the interface which is allowed to cut through the element. They have proved optimal order of convergence in the $H^{1}$ - and $L^{2}$-norms. Latige et al. presented second order accurate finite volume method for interface problem with discontinuous coefficients in [16]. This paper is a generalization of one-dimensional work given in [8] to two-dimensional elliptic interface problems including curve boundaries.

In this paper, we first derive weak formulation of second order elliptic interface problem along with jump conditions. Then we give an algorithm to solve numerically these problems in the simple domain, namely, rectangle with straight line interface. We generalize the algorithm for domain with a curved boundary and interface by using a transformation socalled Gordon and Hall map. For a complicated domain, the spectral element collocation method is adopted by decomposing the domain into some nonoverlapping subdomains and then the spectral collocation method is used in each subdomain. Finally some numerical experiments are given to show efficiency and accuracy of our method.

\section{Pseudo-Spectral Collocation Method}

In this section we present an algorithm for solving an elliptic interface problem by the spectral collocation method. First we start with an elliptic interface problem with a discontinuous constant coefficient and a singular source term defined on a simple domain with line interface and then we extend it to an elliptic interface problem with any arbitrary domain and interface. We review some simple facts including function spaces, interpolation operators, and spectral matrices for the use of the spectral collocation method from now on. The standard notations and definitions are used for the weighted Sobolev spaces $H_{\omega}^{s}(\Omega)$ equipped with weighted inner product $(\cdot, \cdot)_{s, \omega}$ and corresponding weighted norms $\|\cdot\|_{s, \omega}, s \geq 0$, where $\omega(x, y)=\widehat{\omega}(x) \widehat{\omega}(y)$ is the Legendre weight function when $\widehat{\omega}(t)=1$ and Chebyshev weight function when $\widehat{\omega}(t)=$ $1 / \sqrt{1-t^{2}}$. Let $\mathscr{P}_{N}$ be the space of all polynomials of degree less than or equal to $N$ and let $\left\{\xi_{i}\right\}_{i=0}^{N}$ be the Legendre Gauss Lobatto (LGL) or Chebyshev Gauss Lobatto (CGL) points on $[-1,1]$ such that $-1=: \xi_{0}<\xi_{1}<\cdots<\xi_{N-1}<\xi_{N}:=1$. For Legendre case, $\left\{\xi_{i}\right\}_{i=0}^{N}$ is the set of zeros of $\left(1-t^{2}\right) L_{N}^{\prime}(t)$, where $L_{N}$ is the $N$ th Legendre polynomial and the corresponding quadrature weights $\left\{w_{i}\right\}_{i=0}^{N}$ are given by

$$
\begin{array}{r}
w_{0}=w_{N}=\frac{2}{N(N+1)}, \quad w_{j}=\frac{2}{N(N+1)} \frac{1}{\left[L_{N}\left(\xi_{j}\right)\right]^{2}}, \\
1 \leq j \leq N-1 .
\end{array}
$$

For Chebyshev case, $\left\{\xi_{i}\right\}_{i=0}^{N}$ is the set of zeros of $(1-$ $\left.t^{2}\right) T_{N}^{\prime}(t)$, where $T_{N}$ is the $N$ th Chebyshev polynomial and the corresponding quadrature weights $\left\{w_{i}\right\}_{i=0}^{N}$ are given by

$$
w_{0}=w_{N}=\frac{\pi}{2 N}, \quad w_{j}=\frac{\pi}{N}, \quad 1 \leq j \leq N-1 .
$$

For any continuous function $u$ on $[-1,1]$, let $I_{N} u$ denote its Lagrange interpolation at collocation points $\left\{\xi_{i}\right\}_{i=0}^{N}$; that is,

$$
I_{N} u\left(\xi_{i}\right)=u\left(\xi_{i}\right), \quad i=0,1 \ldots, N
$$

Let $\left\{\psi_{j}\right\}_{j=0}^{N} \subset \mathscr{P}_{N}$ be the Lagrange basis functions of degree $N$ such that

$$
\psi_{j}\left(\xi_{k}\right)=\delta_{j k} \quad \forall j, k=0,1, \ldots, N
$$

Then

$$
I_{N} u(x)=\sum_{j=0}^{N} u\left(\xi_{j}\right) \psi_{j}(x) .
$$

The pseudo-spectral derivative $\partial_{N} u$ of a continuous function $u$ is defined to be the exact derivative of the interpolant of $u$; that is,

$$
\partial_{N} u\left(\xi_{i}\right)=\sum_{j=0}^{N} \psi_{j}^{\prime}\left(\xi_{i}\right) u\left(\xi_{j}\right)
$$

Then the pseudo-spectral derivative matrix $D_{N}$ is

$$
D_{N}(i, j):=\psi_{j}^{\prime}\left(\xi_{i}\right)
$$

Let $U$ be the vector valued function containing nodal values of $u$ at $\xi_{j}$; that is, $U=\left(u\left(\xi_{0}\right), \ldots, u\left(\xi_{N}\right)\right)^{T}$; then the derivative vector valued function of $u^{\prime}$ is $D_{N} U=\left(u^{\prime}\left(\xi_{0}\right), \ldots, u^{\prime}\left(\xi_{N}\right)\right)^{T}$. If the interval $[-1,1]$ is replaced by $[a, b]$, we can use the following linear transformation:

$$
t=\frac{b-a}{2}(x+1)+a:[-1,1] \longrightarrow[a, b]
$$

to find Gauss-points $\left\{\widehat{\xi}_{j}\right\}_{j=0}^{N}$ and the quadrature weights $\left\{\widehat{w}_{j}\right\}_{j=1}^{N}$

$$
\widehat{\xi}_{j}=\frac{b-a}{2}\left(\xi_{j}+1\right)+a, \quad \widehat{w}_{j}=\frac{b-a}{2} w_{j} .
$$

Introducing

$$
\widehat{\psi}_{j}(t):=\psi_{j}(x)=\psi_{j}\left(\frac{2}{b-a}(t-a)-1\right)
$$

we easily obtain the following spectral matrix $\widehat{D}_{N}$

$$
\widehat{D}_{N}=\frac{2}{b-a} D_{N} \quad \text { by } \widehat{D}_{N}(i, j):=\widehat{\psi}_{j}^{\prime}\left(\widehat{\xi}_{i}\right)=\frac{2}{b-a} \psi_{j}^{\prime}\left(\xi_{i}\right) \text {. }
$$


The two-dimensional LGL and CGL nodes $\left\{\mathbf{x}_{i j}\right\}$ and weights $\left\{w_{i j}\right\}$ are defined as

$$
\mathbf{x}_{i j}=\left(\xi_{i}, \xi_{j}\right), \quad w_{i j}=w_{i} w_{j}, \quad i, j=0,1, \ldots, N .
$$

Let $\mathbb{Q}_{N}$ be the space of polynomials of degree less than or equal to $N$ with respect to each variable $x$ and $y$. The basis functions are also defined

$$
\psi_{i j}(x, y)=\psi_{i}(x) \psi_{j}(y), \quad i, j=0,1, \ldots, N .
$$

We reorder the LGL and CGL points from bottom to top and then from left to right such that $\mathbf{x}_{j}$ for $j=1, \ldots,(N+1)^{2}$. Then pseudo-spectral derivative matrix on 2-dimensional space is defined via the Kronecker tensor product; that is,

$$
\begin{array}{cl}
S_{x}=D_{N} \otimes I_{N}, & S_{y}=I_{N} \otimes D_{N}, \\
S_{x x}=D_{N}^{2} \otimes I_{N}, & S_{y y}=I_{N} \otimes D_{N}^{2},
\end{array}
$$

where $I_{N}$ is identity matrix of the same order as $D_{N}$.

Let $\Omega=(a, b) \times(c, d)$ be a quadrilateral domain and let $\Gamma=\{\alpha\} \times(c, d)$ be an interface separating the domain $\Omega$ into two subdomains $\Omega^{+}$and $\Omega^{-}$, such that $\bar{\Omega}=\overline{\Omega^{+}} \cup \overline{\Omega^{-}} \cup \Gamma$. Here, $\Gamma$ is referred to as an interface. The boundary of $\Omega$ is denoted by $\partial \Omega$ and $\partial \Omega^{+}=\overline{\Omega^{+}} \cap \partial \Omega, \partial \Omega^{-}=\overline{\Omega^{-}} \cap \partial \Omega$. Consider the following elliptic interface problem:

$$
\begin{gathered}
-\nabla \cdot(\beta \nabla u)+k u=f+g \delta_{\Gamma} \quad \text { in } \Omega, \\
u=0 \quad \text { on } \partial \Omega,
\end{gathered}
$$

where $g \in C(\Gamma), f \in L^{2}(\Omega)$ and $\beta, k \in L^{\infty}(\Omega)$, with

$$
\begin{aligned}
& \beta(x, y)= \begin{cases}\beta^{+}(x, y) & (x, y) \in \Omega^{+} \\
\beta^{-}(x, y) & (x, y) \in \Omega^{-},\end{cases} \\
& k(x, y)= \begin{cases}\kappa^{+}(x, y) & (x, y) \in \Omega^{+} \\
\kappa^{-}(x, y) & (x, y) \in \Omega^{-},\end{cases}
\end{aligned}
$$

and $\delta_{\Gamma}$ is a 2-dimensional delta function with the support along the interface $\Gamma$.

In order to solve this problem via the interface problem, we need to first derive the jump conditions. It is obvious from the given equation that there exists one jump discontinuity by the Dirac $\delta$-function. Using the standard finite element argument $[6,9]$, we have the following classical formulation:

$$
\begin{gathered}
-\nabla \cdot\left(\beta \nabla u^{ \pm}\right)+k u^{ \pm}=f^{ \pm} \quad \text { in } \Omega^{ \pm}, \\
u^{ \pm}=0 \quad \text { on } \partial \Omega^{ \pm},
\end{gathered}
$$

along with the following interface jump conditions:

$$
\left.[u]\right|_{\Gamma}=0,\left.\quad[\beta \nabla u \cdot \mathbf{n}]\right|_{\Gamma}=g,
$$

where $\mathbf{n}=\left(n_{1}, n_{2}\right)$ denotes the outward normal vector on $\partial \Omega^{+}$. The interface jump is defined as follows:

$$
\left.[v]\right|_{\Gamma}=v^{+}-v^{-},
$$

where $v^{+}$and $v^{-}$are the traces of $\left.v\right|_{\Omega^{+}}$and $\left.v\right|_{\Omega^{-}}$, respectively, on $\Gamma$. The existence and uniqueness of solution of (17) is studied in [17].

Here we consider the pseudo-spectral method for the problem (17)-(18) with $\beta$ and $\kappa$ as piecewise continuous constants. Suppose that $f$ is a piecewise continuous function and $g$ is continuous on $\Gamma$. Suppose that $u_{N} \in \mathbb{Q}_{N}$ is the pseudo-spectral approximation solution to problem (17)-(18). Then the approximation solution of the interface problem (17) can be expressed by

$$
u^{ \pm}(x, y)=\sum_{i=0}^{N} \sum_{j=0}^{N} u_{i j}^{ \pm} \psi_{i j}^{ \pm}(x, y) .
$$

Although it is possible to use different polynomial order approximation on each subdomain, we use the same polynomial order approximation, for the sake of simplicity. Hence we have the following equations:

$$
\begin{array}{r}
-\beta^{ \pm} \Delta u^{ \pm}\left(\xi_{i}, \xi_{j}\right)+\kappa^{ \pm} u^{ \pm}\left(\xi_{i}, \xi_{j}\right)=f^{ \pm}\left(\xi_{i}, \xi_{j}\right) \\
\forall\left(\xi_{i}, \xi_{j}\right) \in \Omega^{ \pm},
\end{array}
$$

which can be written in matrix-vector form as

$$
\begin{aligned}
A_{1} U= & {\left[\begin{array}{l|l}
-\beta^{+}\left(S_{x x}^{+}+S_{y y}^{+}\right)+\kappa^{+} I & \mathbf{0} \\
\hline \mathbf{0} & -\beta^{-}\left(S_{x x}^{-}+S_{y y}^{-}\right)+\kappa^{-} I
\end{array}\right] } \\
& \times\left[\frac{U^{+}}{U^{-}}\right] \\
= & {\left[\frac{F^{+}}{F^{-}}\right]=F_{1}, }
\end{aligned}
$$

where $U^{ \pm}=\left(u^{ \pm}\left(\mathbf{x}_{i}\right)\right)$ and $F^{ \pm}=\left(f^{ \pm}\left(\mathbf{x}_{i}\right)\right)$. Here

$$
\begin{array}{ll}
S_{x x}^{+}=\left(\frac{2}{\alpha-a}\right)^{2} D_{N}^{2} \otimes I_{N}, & S_{y y}^{+}=\left(\frac{2}{d-c}\right)^{2} I_{N} \otimes D_{N}^{2}, \\
S_{x x}^{-}=\left(\frac{2}{b-\alpha}\right)^{2} D_{N}^{2} \otimes I_{N}, & S_{y y}^{-}=\left(\frac{2}{d-c}\right)^{2} I_{N} \otimes D_{N}^{2} .
\end{array}
$$

Let $U^{ \pm}=\left[\begin{array}{lll}U_{\mathrm{bd}}^{ \pm} & U_{\text {in }}^{ \pm} & U_{\mathrm{if}}^{ \pm}\end{array}\right]^{t}$, where $U_{\mathrm{if}}^{ \pm}, U_{\mathrm{bd}}^{ \pm}$, and $U_{\text {in }}^{ \pm}$denote by the values of $u^{ \pm}$at nodal points on the interface, boundaries, and interior of domain $\Omega^{ \pm}$, respectively. By jump conditions $\left.[u]\right|_{\Gamma}=0$ and $\left.[\beta \nabla u \cdot \mathbf{n}]\right|_{\Gamma}=g$, we have $u^{+}-u^{-}=0$ and $\beta^{+} \nabla u^{+} \cdot \mathbf{n}-\beta^{-} \nabla u^{-} \cdot \mathbf{n}=g$, respectively, which can be written in matrix-vector form as

$$
\begin{gathered}
U_{\mathrm{if}}^{+}-U_{\mathrm{if}}^{+}=0, \\
\left(\beta^{+} S_{x}^{+} \cdot n_{1}+\beta^{+} S_{y}^{+} \cdot n_{2}\right) U_{\mathrm{if}}^{+} \\
-\left(\beta^{-} S_{x}^{-} \cdot n_{1}+\beta^{-} S_{y}^{-} \cdot n_{2}\right) U_{\mathrm{if}}^{-}=G,
\end{gathered}
$$

where $G=\left(g\left(\mathbf{x}_{i}\right)\right)$ for $\mathbf{x}_{i}$ on $\Gamma$. The boundary conditions can be imposed as

$$
U_{\mathrm{bd}}^{ \pm}=0 .
$$


Now, the boundary and jump conditions can be represented as

$$
\begin{aligned}
& \mathrm{A}_{2} \mathrm{U} \\
& \times\left[\begin{array}{c}
U_{\text {bd }}^{+} \\
U_{\text {in }}^{+} \\
U_{\text {if }}^{+} \\
\hline U_{\text {bd }}^{-} \\
U_{\text {in }}^{-} \\
U_{\text {if }}^{-}
\end{array}\right]=\left[\begin{array}{c}
0 \\
0 \\
0 \\
\hline 0 \\
0 \\
G
\end{array}\right]=F_{2} .
\end{aligned}
$$$$
=\left[\begin{array}{ccc|ccc}
I & 0 & 0 & 0 & 0 & 0 \\
0 & 0 & 0 & 0 & 0 & 0 \\
0 & 0 & I & 0 & 0 & -I \\
\hline 0 & 0 & 0 & I & 0 & 0 \\
0 & 0 & 0 & 0 & 0 & 0 \\
0 & 0 & \beta^{+} S_{x}^{+} n_{1}+\beta^{+} S_{y}^{+} n_{2} & 0 & 0 & -\beta^{-} S_{x}^{-} n_{1}-\beta^{-} S_{y}^{-} n_{2}
\end{array}\right]
$$

We also note that

$$
\begin{array}{ll}
S_{x}^{+}=\left(\frac{2}{\alpha-a}\right) D_{N} \otimes I_{N}, & S_{y}^{+}=\left(\frac{2}{d-c}\right) I_{N} \otimes D_{N}, \\
S_{x}^{-}=\left(\frac{2}{b-\alpha}\right) D_{N} \otimes I_{N}, & S_{y}^{-}=\left(\frac{2}{d-c}\right) I_{N} \otimes D_{N} .
\end{array}
$$

Combining two systems, we have the following linear system:

$$
A U=F,
$$

where $A=A_{1}+A_{2}$ and $F=F_{1}+F_{2}$. The resulting algebraic system can be efficiently solved by direct and iterative methods.

We now generalize the above algorithm for the case of discontinuous varying functions $\beta$ and $k$. We still assume that the domain $\Omega$ is rectangle and the interface is straight line as before. In this case, the first equation in (17) can be expanded as follows:

$$
-\beta^{ \pm} \Delta u^{ \pm}+\nabla \beta^{ \pm} \cdot \nabla u^{ \pm}+k^{ \pm} u^{ \pm}=f \quad \text { in } \Omega^{ \pm}
$$

Hence the pseudo-spectral method for (29) reads:

$$
\begin{aligned}
& -\beta^{ \pm}\left(\xi_{i}, \xi_{j}\right) \Delta u^{ \pm}\left(\xi_{i}, \xi_{j}\right)+\nabla \beta^{ \pm}\left(\xi_{i}, \xi_{j}\right) \cdot \nabla u^{ \pm}\left(\xi_{i}, \xi_{j}\right) \\
& \quad+k^{ \pm}\left(\xi_{i}, \xi_{j}\right) u^{ \pm}\left(\xi_{i}, \xi_{j}\right) \\
& \quad=f\left(\xi_{i}, \xi_{j}\right) \\
& \forall\left(\xi_{i}, \xi_{j}\right) \in \Omega^{ \pm},
\end{aligned}
$$

which can be written in matrix-vector form as

$$
A_{1} U=F_{1}
$$

with

$$
A_{1}=\left[\begin{array}{c|c}
-\boldsymbol{\beta}^{+}\left(S_{x x}^{+}+S_{y y}^{+}\right) & \mathbf{0} \\
+\boldsymbol{\beta}_{x}^{+} S_{x}^{+}+\boldsymbol{\beta}_{y}^{+} S_{y}^{+} & \\
+\mathbf{k}^{+} I & -\boldsymbol{\beta}^{-}\left(S_{x x}^{-}+S_{y y}^{-}\right) \\
& +\boldsymbol{\beta}_{x}^{-} S_{x}^{-}+\boldsymbol{\beta}_{y}^{-} S_{y}^{-} \\
& +\mathbf{k}^{-} I
\end{array}\right],
$$

$U=\left[\begin{array}{ll}U^{+} & U^{-}\end{array}\right]^{t}$ and $F_{1}=\left[\begin{array}{ll}I_{N} f^{+} & I_{N} f^{-}\end{array}\right]^{t}$, where $\boldsymbol{\beta}^{ \pm}, \boldsymbol{\beta}_{x}^{ \pm}, \boldsymbol{\beta}_{y}^{ \pm}$ and $\mathbf{k}^{ \pm}$are diagonal matrices of functions $\beta, \beta_{x}, \beta_{y}$, and $k$ evaluated at nodal points $\left(\xi_{i}, \xi_{j}\right) \in \Omega^{ \pm}$, respectively. In this case, the boundary and interface conditions remain unchanged.

The algorithm proposed before is to solve interface elliptic equations defined in a quadrilateral domain with straight line interface. One may generalize the algorithm to solve interface problems defined in a domain with curved boundaries using Gordon and Hall transformation [18, 19]. Here we briefly introduce the Gordon and Hall transformation on a simply connected domain $\Omega$ for reader's convenience (see $[18,19]$ for more details.).

Let $\mathbf{F}$ be a vector-valued function of two independent variables $\widehat{x}$ and $\hat{y}$ over a domain $\mathbf{S}=[0, h] \times[0, h]$ in the $\hat{x} \hat{y}$-plane whose range is $\Omega$ in $\mathbb{R}^{2}$. We assume that $\mathbf{F}$ is a continuous one-to-one transformation which maps $\mathbf{S}$ onto the domain $\bar{\Omega}$ such that $\mathbf{F}: \partial \mathbf{S} \rightarrow \partial \Omega$. Then we would like to construct a one-to-one function $\mathbf{T}: \mathbf{S} \rightarrow \Omega$ which matches $\mathbf{F}$ on the boundaries of $\mathbf{S}$, so-called the boundary interpolant of $F$ :

$$
\begin{array}{lll}
\mathbf{T}(0, \hat{y})=\mathbf{F}(0, \hat{y}), & \mathbf{T}(h, \widehat{y})=\mathbf{F}(h, \widehat{y}), & 0 \leq \widehat{y} \leq h, \\
\mathbf{T}(\widehat{x}, 0)=\mathbf{F}(\widehat{x}, 0), & \mathbf{T}(\widehat{x}, h)=\mathbf{F}(\widehat{x}, h), & 0 \leq \widehat{x} \leq h .
\end{array}
$$

Following the ideas given in $[18,19]$, one may choose the following simple transfinite bilinear Lagrange interpolant of F:

$$
\begin{aligned}
\mathbf{T}(\hat{x}, \hat{y})= & {\left[\begin{array}{l}
x(\hat{x}, \widehat{y}) \\
y(\hat{x}, \widehat{y})
\end{array}\right] } \\
:= & \left(1-\frac{\widehat{x}}{h}\right) \mathbf{F}(0, \widehat{y})+\left(\frac{\widehat{x}}{h}\right) \mathbf{F}(h, \widehat{y}) \\
& +\left(1-\frac{\widehat{y}}{h}\right) \mathbf{F}(\widehat{x}, 0)+\left(\frac{\widehat{y}}{h}\right) \mathbf{F}(\widehat{x}, h) \\
& -\left(1-\frac{\widehat{x}}{h}\right)\left(1-\frac{\widehat{y}}{h}\right) \mathbf{F}(0,0) \\
& -\left(1-\frac{\widehat{x}}{h}\right)\left(\frac{\widehat{y}}{h}\right) \mathbf{F}(0, h) \\
& -\left(1-\frac{\widehat{y}}{h}\right)\left(\frac{\hat{x}}{h}\right) \mathbf{F}(h, 0) \\
& -\left(\frac{\hat{y}}{h}\right)\left(\frac{\widehat{x}}{h}\right) \mathbf{F}(h, h) .
\end{aligned}
$$


Then we have the transformed function $\widehat{u}(\widehat{x}, \widehat{y}):=u(\mathbf{T}(\widehat{x}, \widehat{y}))$ defined on the rectangular domain $\mathbf{S}$ and the elliptic equation defined on the curved domain $\Omega$ is also transformed to an elliptic equation defined on the rectangular domain $\mathbf{S}$ so that we can apply our proposed method to the transformed equation. The transformed equation can be represented as

$$
-a^{ \pm} \Delta \widehat{u}^{ \pm}+\mathbf{b}^{ \pm} \cdot \nabla \widehat{u}^{ \pm}+c^{ \pm} \widehat{u}^{ \pm}=\widehat{f}^{ \pm} \quad \text { in } \mathbf{S}^{ \pm}
$$

whereas the coefficients $a^{ \pm}, \mathbf{b}^{ \pm}=\left(b_{1}^{ \pm}, b_{2}^{ \pm}\right)$, and $c^{ \pm}$are functions in $\hat{x}$ and $\hat{y}$. Applying the spectral collocation method to the above equations we have the following matrix form:

$$
A_{1}=\left[\begin{array}{c|c}
-\widehat{\mathbf{a}}^{+}\left(S_{x x}^{+}+S_{y y}^{+}\right) & \\
+\widehat{\mathbf{b}}_{1}^{+} S_{x}+\widehat{\mathbf{b}}_{2}^{+} S_{y} & \mathbf{0} \\
+\widehat{\mathbf{c}}^{+} I & -\widehat{\mathbf{a}}^{-}\left(S_{x x}^{-}+S_{y y}^{-}\right) \\
& +\widehat{\mathbf{b}}_{1}^{-} S_{x}+\widehat{\mathbf{b}}_{2}^{-} S_{y} \\
& +\widehat{\mathbf{c}}^{-} I
\end{array}\right],
$$

where $\widehat{\mathbf{a}}^{ \pm}, \widehat{\mathbf{b}}_{1}^{ \pm}, \widehat{\mathbf{b}}_{2}^{ \pm}$, and $\widehat{\mathbf{c}}^{ \pm}$are diagonal matrices of corresponding function values evaluated at nodal points $\mathbf{x}_{i} \in \mathbf{S}^{ \pm}$, respectively. The jump and boundary conditions are similarly given as the previous $A_{2}$. For complex geometries we employ pseudo-spectral element method. First, we decompose the domain $\Omega$ into some nonoverlapping subdomains and then apply the spectral collocation method for each subdomain.

Finally, we give an example of the Gordon-Hall transformation for the domain given in Figure 2. The boundary interpolant is given by

$$
\begin{aligned}
\mathbf{F}(\widehat{x}, 0)=\left(\begin{array}{c}
\hat{x} \\
-\frac{\hat{x}}{4}
\end{array}\right), \quad \mathbf{F}(\widehat{x}, h)=\left(\begin{array}{c}
\widehat{x} \\
\frac{\hat{x}}{4}+2
\end{array}\right), \quad 0 \leq \hat{x} \leq 2, \\
\mathbf{F}(h, \hat{y})=\left(\begin{array}{c}
2 \\
\frac{3}{2} \hat{y}-\frac{1}{2}
\end{array}\right), \\
\mathbf{F}(0, \hat{y})=\left(\begin{array}{c}
\cos \left(\frac{(\widehat{y}-1) \pi}{2}\right) \\
1+\sin \left(\frac{(\hat{y}-1) \pi}{2}\right)
\end{array}\right), \\
0 \leq \hat{y} \leq 2,
\end{aligned}
$$

and the explicit form of the transformation in (34) reduces to

$$
\mathbf{T}(\hat{x}, \hat{y})=\left[\begin{array}{c}
\left(1-\frac{\hat{x}}{2}\right) \cos \left(\frac{(\hat{y}-1) \pi}{2}\right)+\hat{x} \\
\left(1-\frac{\hat{x}}{2}\right) \sin \left(\frac{(\hat{y}-1) \pi}{2}\right)+1-\frac{3}{4} \hat{x}+\frac{3}{4} \widehat{y} \hat{x}
\end{array}\right] .
$$

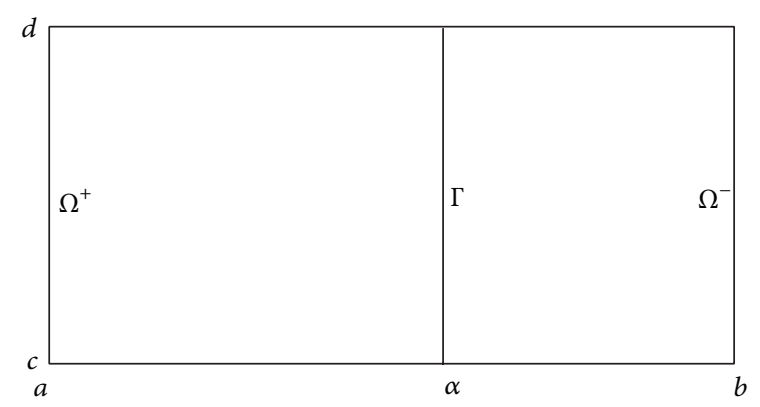

Figure 1: Interface domain.

$(2,2.5)$

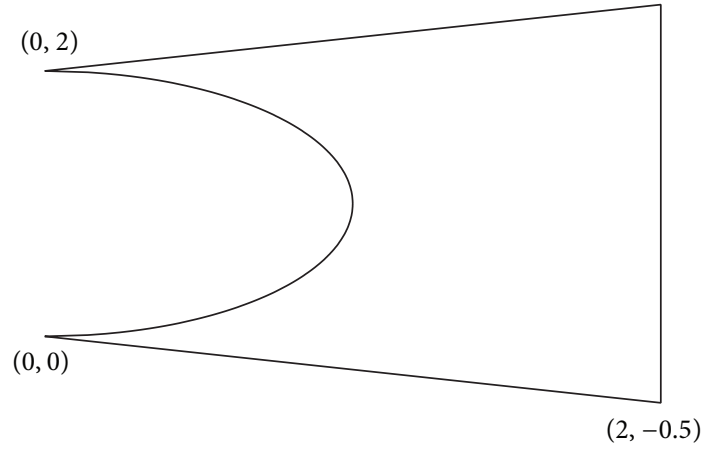

FIGURE 2: A curved domain.

\section{Numerical Results}

In this section, we first give an example defined on rectangle domain with straight line interface as in Figure 1. Afterward examples are defined on more complicated domain in which we have to use spectral element collocation method to solve them. Denote by $u_{N}$ the discrete solution of interface problem and by $e=u-u_{N}$, its errors. We present their $L_{w}^{2}(\Omega)$ and $H_{w}^{1}(\Omega)$ discrete norm which is defined, respectively, as follows:

$$
\begin{gathered}
\|e\|_{0, w, N}^{2}=\sum_{i, j=0}^{N} w_{i j} e^{2}\left(\mathbf{x}_{i j}\right), \\
\|e\|_{1, w, N}^{2}=\|\nabla e\|_{0, w, N}^{2}+\|e\|_{0, w, N}^{2} .
\end{gathered}
$$

Example 1 (straight line interface). Consider

$$
\begin{gathered}
\beta u_{x x}+u_{y y}+u=f+g \delta_{\alpha}, \quad \text { in } \Omega=(0, L) \times(0,1), \\
u=0 \quad \text { on } \partial \Omega,
\end{gathered}
$$

with the following exact solution:

$$
\begin{aligned}
& u(x, y) \\
& =\sin (\pi y) \\
& \quad \cdot\left\{\begin{array}{c}
C_{1} \cos \left(x \gamma_{-}\right)+C_{2} \sin \left(x \gamma_{-}\right)+1, \\
\text { in } \Omega_{-}=(0, \alpha) \times(0,1), \\
C_{3} \cos \left(x \gamma_{+}\right)+C_{4} \sin \left(x \gamma_{+}\right)+1, \\
\text { in } \Omega_{+}=(\alpha, L) \times(0,1),
\end{array}\right.
\end{aligned}
$$




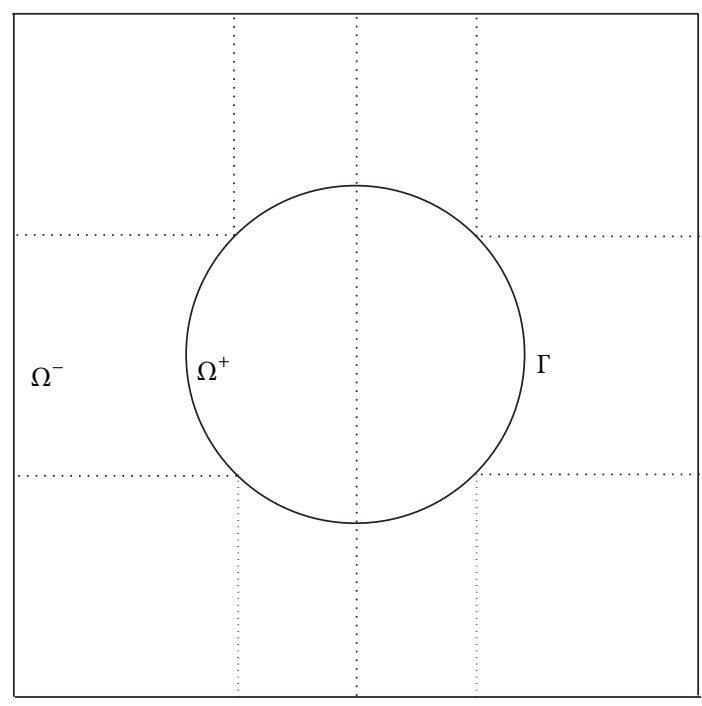

FIgURE 3: Domain decomposition for the interface examples.

TABLE 1: Error discretization for Example 1 with $v=0$.

\begin{tabular}{lcccc}
\hline \multirow{2}{*}{$N$} & \multicolumn{2}{c}{ Legendre } & \multicolumn{2}{c}{ Chebyshev } \\
& $\|e\|_{0, w, N}$ & $\|e\|_{1, w, N}$ & $\|e\|_{0, w, N}$ & $\|e\|_{1, w, N}$ \\
\hline 4 & $1.0355 e-003$ & $3.5826 e-003$ & $7.6340 e-003$ & $2.7116 e-002$ \\
8 & $2.0795 e-008$ & $3.1680 e-007$ & $7.4546 e-007$ & $1.5090 e-005$ \\
12 & $1.7134 e-013$ & $4.5879 e-012$ & $1.4805 e-011$ & $6.4867 e-010$ \\
16 & $7.9221 e-016$ & $8.9314 e-015$ & $4.6954 e-015$ & $4.2893 e-014$ \\
20 & $2.0792 e-015$ & $2.5554 e-014$ & $1.5121 e-014$ & $1.2825 e-013$ \\
\hline
\end{tabular}

where

$$
\begin{array}{cc}
\gamma_{+}=\frac{1}{\sqrt{10}}, & \gamma_{-}=\frac{1}{10}, \\
\alpha=\frac{\sqrt{10} \pi}{6}, & L=\frac{\sqrt{10} \pi}{2}, \\
\beta^{-}=100, & \beta^{+}=10,
\end{array}
$$

and $C_{i}$ 's can be determined by the boundary and jump conditions. We have

$$
g=\nu \sin (\pi y)
$$

$$
\begin{aligned}
f=\sin (\pi y) \\
\cdot \begin{cases}1-\pi^{2}\left(C_{1} \cos \left(x \gamma_{-}\right)+C_{2} \sin \left(x \gamma_{-}\right)+1\right), & \text { in } \Omega_{-}, \\
1-\pi^{2}\left(C_{3} \cos \left(x \gamma_{+}\right)+C_{4} \sin \left(x \gamma_{+}\right)+1\right), & \text { in } \Omega_{+} .\end{cases}
\end{aligned}
$$

Tables 1 and 2 show the spectral convergence in $L_{\omega}^{2}(\Omega)$ and $H_{\omega}^{1}(\Omega)$-norm errors of Legendre and Chebyshev approximation for $\nu=0$ and $\nu=10$, respectively.

As we pointed out in Section 2, it is possible to use different polynomial order approximation on each subdomain. To
TABLE 2: Error discretization for Example 1 with $v=10$.

\begin{tabular}{lcccc}
\hline \multirow{2}{*}{$N$} & \multicolumn{2}{c}{ Legendre } & \multicolumn{2}{c}{ Chebyshev } \\
& $\|e\|_{0, w, N}$ & $\|e\|_{1, w, N}$ & $\|e\|_{0, w, N}$ & $\|e\|_{1, w, N}$ \\
\hline 4 & $5.5773 e-004$ & $1.8956 e-003$ & $2.9508 e-003$ & $1.0608 e-002$ \\
8 & $1.1177 e-008$ & $1.6996 e-007$ & $4.4191 e-007$ & $9.3991 e-006$ \\
12 & $9.6555 e-014$ & $2.6250 e-012$ & $9.2719 e-012$ & $4.4550 e-010$ \\
16 & $1.9355 e-015$ & $2.2839 e-014$ & $1.4037 e-014$ & $1.2251 e-013$ \\
20 & $5.8201 e-015$ & $6.8838 e-014$ & $3.8994 e-014$ & $3.2236 e-013$ \\
\hline
\end{tabular}

TABLE 3: Error discretization for Example 1 with $\nu=0$ and different polynomial order approximation.

\begin{tabular}{lcccc}
\hline \multirow{2}{*}{$N$} & \multicolumn{2}{c}{ Legendre } & \multicolumn{2}{c}{ Chebyshev } \\
& $\|e\|_{0, w, N}$ & $\|e\|_{1, w, N}$ & $\|e\|_{0, w, N}$ & $\|e\|_{1, w, N}$ \\
\hline 4 & $4.9405 e-06$ & $1.7071 e-05$ & $6.9900 e-06$ & $3.0687 e-05$ \\
8 & $3.9907 e-11$ & $8.5880 e-10$ & $7.8331 e-11$ & $1.8586 e-09$ \\
12 & $9.2143 e-16$ & $3.0729 e-14$ & $1.3772 e-15$ & $4.9032 e-14$ \\
16 & $1.1960 e-15$ & $5.2617 e-14$ & $2.7186 e-15$ & $1.4533 e-13$ \\
20 & $8.8007 e-16$ & $1.6103 e-14$ & $2.9963 e-15$ & $1.8952 e-14$ \\
\hline
\end{tabular}

do this for Example 1, we divide $(0, \alpha)$ into $\left(N_{1}+1\right)$ intervals, $(\alpha, L)$ into $\left(N_{2}+1\right)$ intervals, and $(0,1)$ into $(M+1)$ intervals corresponding to LGL or CGL points. For sake of simplicity we take $N_{1}=N_{2}=N, M=N+2$. The numerical result is given in Table 3 when $v=0$. We have similar results for $\nu \neq 0$. When the interface point $\alpha$ is close to the endpoint, different polynomial order can be used to get accurate results.

In the following we present some numerical experiments in which the domain is $\Omega=\left(\begin{array}{ll}-1 & 1\end{array}\right)^{2}$ and interface $\Gamma$ is the circle $x^{2}+y^{2}=1 / 4$. To use spectral element collocation method, we decompose the domain $\Omega$ into twelve nonoverlapping subdomains as in Figure 3. It should be noted that there is no jump in solution $u$ and its normal derivative for the interface which is different from $\Gamma$; that is,

$$
\left.[u]\right|_{\Gamma^{\prime}}=0,\left.\quad[\beta \nabla u \cdot \mathbf{n}]\right|_{\Gamma^{\prime}}=0,
$$

where $\Gamma^{\prime}$ is dotted line in Figure 3.

Example 2 (circular interface 1 [6]). Consider the elliptic equation of the form

$$
u_{x x}+u_{y y}=2 \delta_{\Gamma} .
$$

In this example, we have only singular source term. The exact solution is given by

$$
u(x, y)= \begin{cases}1, & r \leq \frac{1}{2} \\ 1+\log (2 r), & r>\frac{1}{2},\end{cases}
$$

where $r=\sqrt{x^{2}+y^{2}}$.

The interface conditions are given by

$$
\left.[u]\right|_{\Gamma}=0,\left.\quad[\nabla u \cdot \mathbf{n}]\right|_{\Gamma}=2 .
$$




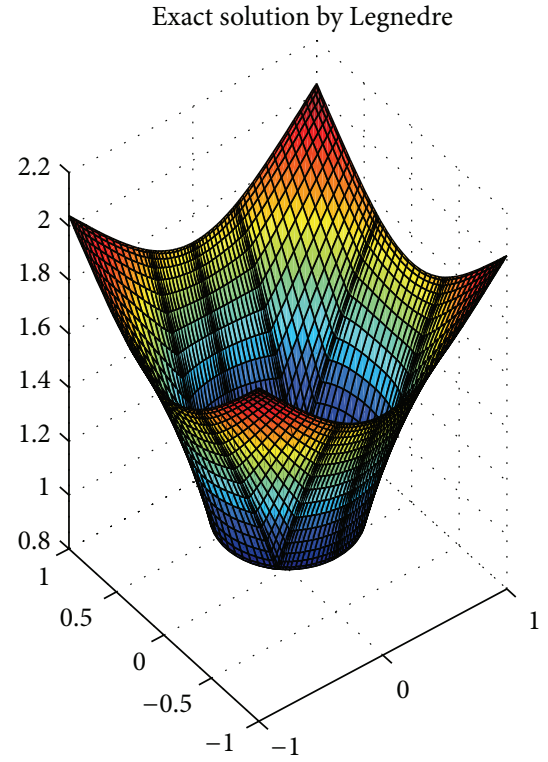

(a)

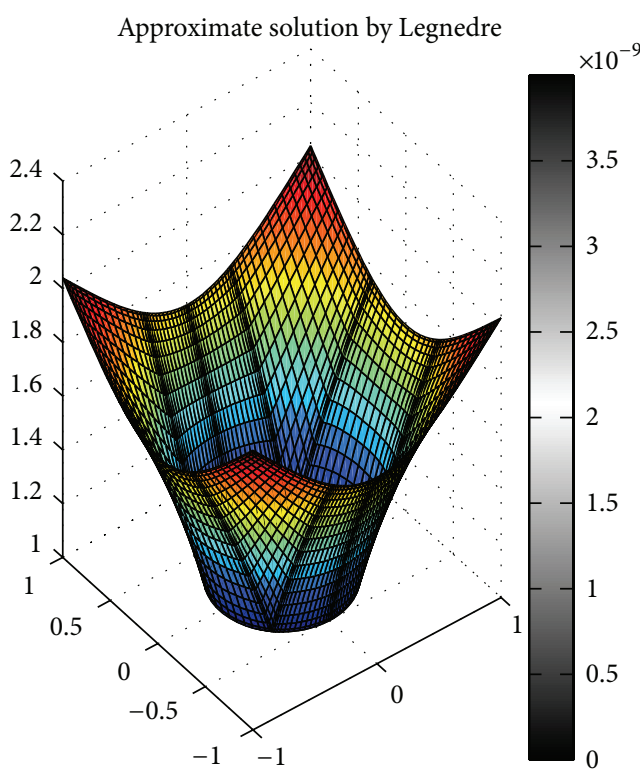

(b)

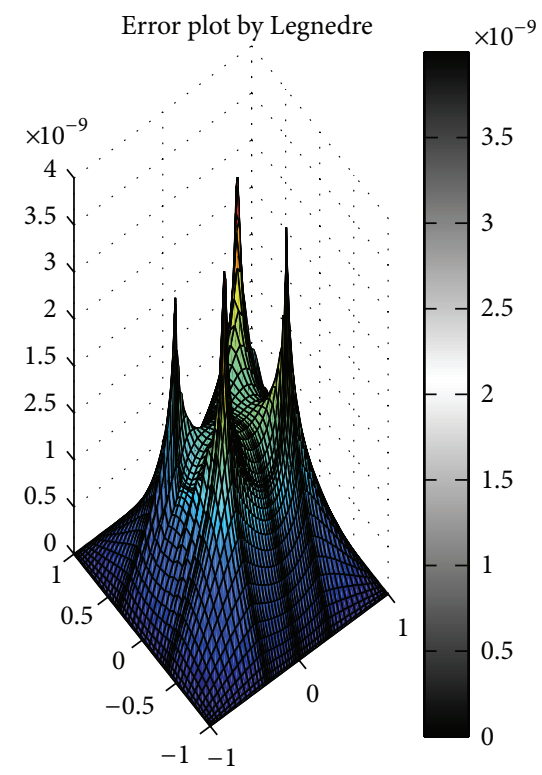

(c)

FIgURE 4: Exact solution, approximation solution, and its error for $N=18$.

Table 4 also shows the spectral convergence in $L_{\omega}^{2}(\Omega)$ - and $H_{\omega}^{1}(\Omega)$-norm errors of Legendre and Chebyshev approximation. The exact solution, approximated one, and its error for the Legendre case of this example are plotted in Figure 4.

Example 3 (circular interface 2 [6]). Consider the elliptic equation

$$
\left(\beta u_{x}\right)_{x}+\left(\beta u_{y}\right)_{y}=f+C \delta_{\Gamma}
$$

where

$$
f=8\left(x^{2}+y^{2}\right)+4
$$

$$
\beta(x, y)= \begin{cases}x^{2}+y^{2}+1, & r \leq \frac{1}{2} \\ b, & r>\frac{1}{2} .\end{cases}
$$




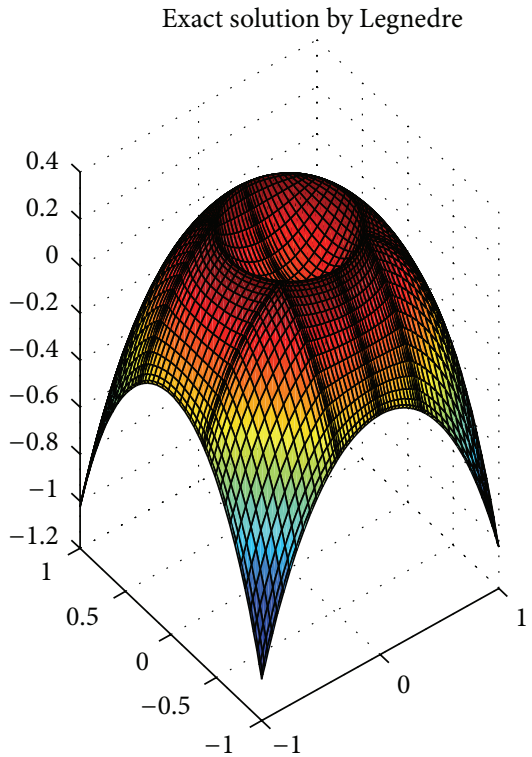

(a)

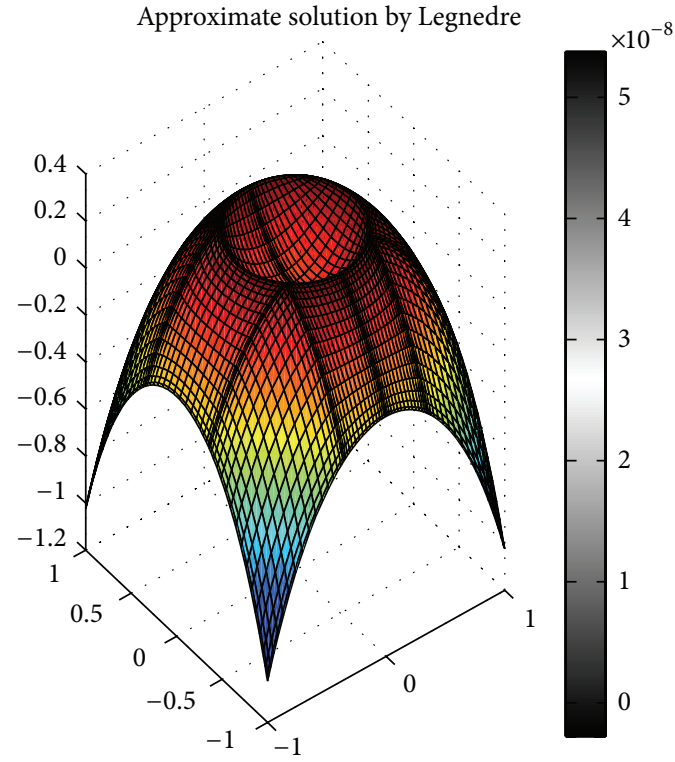

(b)

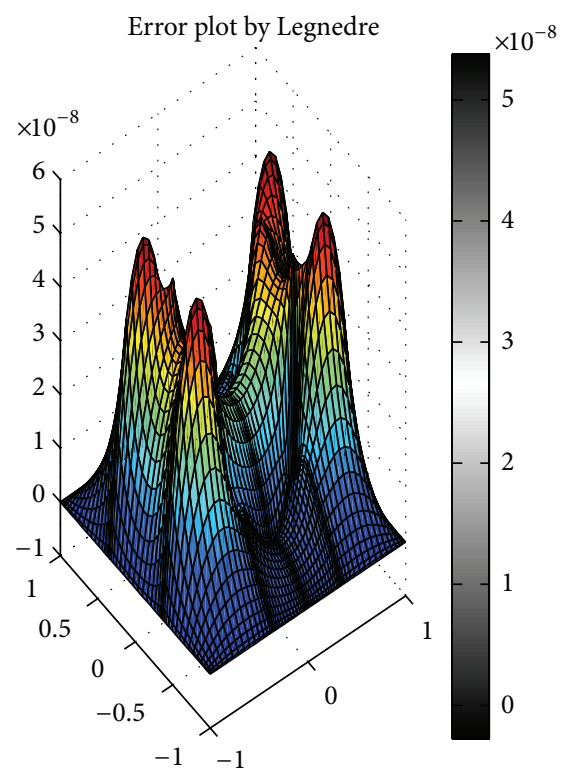

(c)

FIGURE 5: Exact solution, approximation solution, and its error for $C=0.1$ and $b=-3$.

TABLE 4: Error discretization for Example 2.

\begin{tabular}{ccccc}
\hline \multirow{2}{*}{$N$} & \multicolumn{2}{c}{ Legendre } & \multicolumn{2}{c}{ Chebyshev } \\
& $\|e\|_{L^{2}}$ & $\|e\|_{H^{1}}$ & $\|e\|_{L^{2}}$ & $\|e\|_{H^{1}}$ \\
\hline 4 & $9.8536 e-003$ & $3.0592 e-002$ & $3.4684 e-002$ & $1.3468 e-001$ \\
8 & $9.1221 e-005$ & $4.0228 e-004$ & $2.8844 e-003$ & $2.7245 e-002$ \\
12 & $1.1707 e-006$ & $4.6568 e-006$ & $1.3341 e-004$ & $4.3786 e-004$ \\
16 & $1.6054 e-008$ & $6.5734 e-008$ & $4.3721 e-006$ & $1.4678 e-005$ \\
20 & $2.6242 e-010$ & $1.1509 e-009$ & $1.4114 e-007$ & $4.8193 e-007$ \\
24 & $4.1610 e-012$ & $2.1953 e-011$ & $4.4900 e-009$ & $1.5529 e-008$ \\
\hline
\end{tabular}

TABLE 5: Error discretization for $C=0.1$ and $b=-3$ for Example 3 .

\begin{tabular}{ccccc}
\hline \multirow{2}{*}{$N$} & \multicolumn{2}{c}{ Legendre } & \multicolumn{2}{c}{ Chebyshev } \\
& $\|e\|_{L^{2}}$ & $\|e\|_{H^{1}}$ & $\|e\|_{L^{2}}$ & $\|e\|_{H^{1}}$ \\
\hline 4 & $1.0914 e-002$ & $6.7241 e-002$ & $1.3623 e-001$ & $8.8947 e-001$ \\
8 & $2.8725 e-004$ & $1.4547 e-003$ & $1.2226 e-003$ & $9.9894 e-003$ \\
12 & $5.8273 e-006$ & $6.2692 e-005$ & $4.1029 e-005$ & $2.8593 e-004$ \\
16 & $2.5146 e-007$ & $1.2094 e-006$ & $1.4853 e-006$ & $1.0346 e-005$ \\
20 & $7.7489 e-009$ & $3.5890 e-008$ & $5.0604 e-008$ & $3.7029 e-007$ \\
24 & $2.3297 e-010$ & $1.0631 e-009$ & $1.6690 e-009$ & $1.2667 e-008$ \\
\hline
\end{tabular}




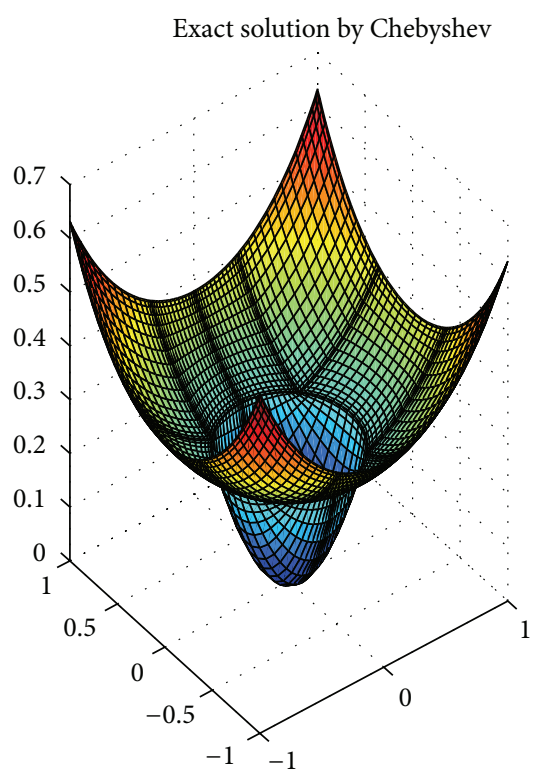

(a)

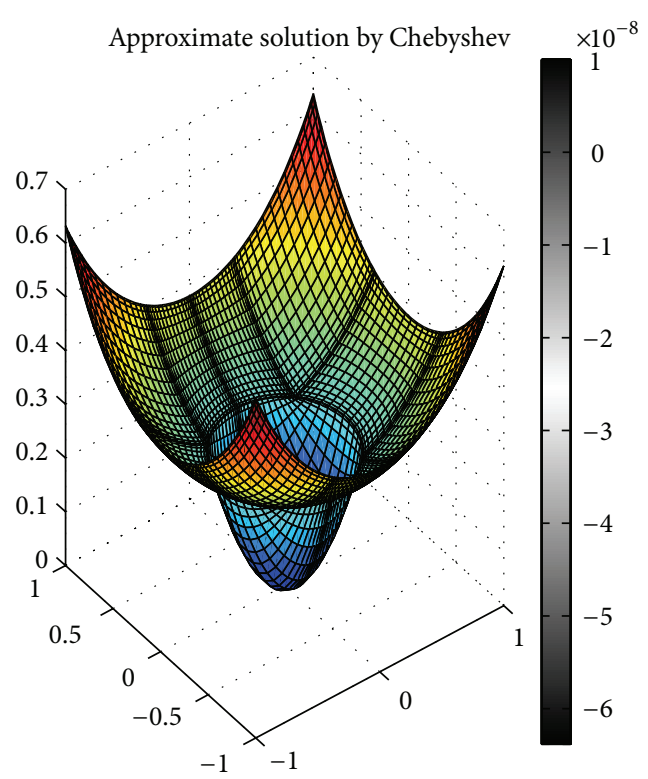

(b)

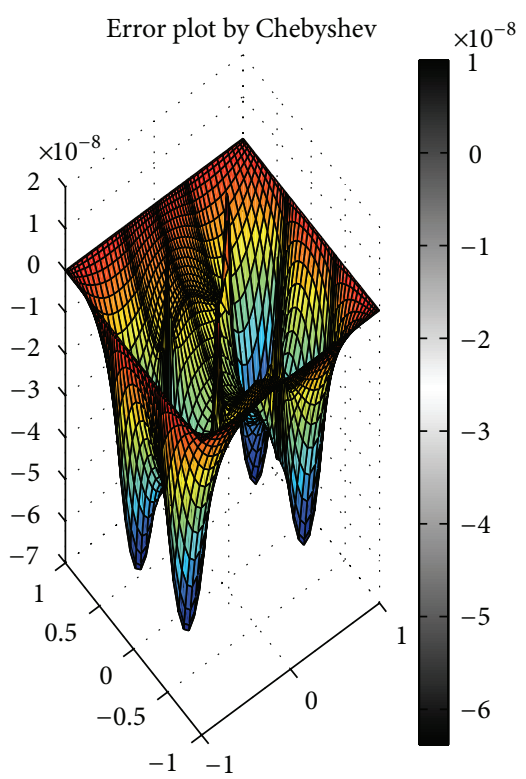

(c)

Figure 6: Exact solution, approximation solution, and its error for $C=0.1$ and $b=10$.

TABLE 6: Error discretization for $C=0.1$ and $b=10$ for Example 3 .

\begin{tabular}{lcccc}
\hline \multirow{2}{*}{$N$} & \multicolumn{2}{c}{ Legendre } & \multicolumn{2}{c}{ Chebyshev } \\
& $\|e\|_{L^{2}}$ & $\|e\|_{H^{1}}$ & $\|e\|_{L^{2}}$ & $\|e\|_{H^{1}}$ \\
\hline 4 & $2.6272 e-003$ & $2.2570 e-002$ & $4.1173 e-002$ & $3.3693 e-001$ \\
8 & $5.8242 e-005$ & $4.0589 e-004$ & $2.8852 e-004$ & $1.9806 e-003$ \\
12 & $2.1978 e-006$ & $1.1109 e-005$ & $1.1563 e-005$ & $7.9872 e-005$ \\
16 & $6.9459 e-008$ & $3.2385 e-007$ & $4.1120 e-007$ & $2.9190 e-006$ \\
20 & $2.1169 e-009$ & $9.5229 e-009$ & $1.3164 e-008$ & $1.1530 e-007$ \\
24 & $6.3490 e-011$ & $2.8077 e-010$ & $4.3819 e-010$ & $3.1670 e-009$ \\
\hline
\end{tabular}

TABLE 7: Error discretization for $C=0.1$ and $b=10$ for Example 4.

\begin{tabular}{lcccc}
\hline \multirow{2}{*}{$N$} & \multicolumn{2}{c}{ Legendre } & \multicolumn{2}{c}{ Chebyshev } \\
& $\|e\|_{L^{2}}$ & $\|e\|_{H^{1}}$ & $\|e\|_{L^{2}}$ & $\|e\|_{H^{1}}$ \\
\hline 4 & $6.8137 e-002$ & $6.3727 e-001$ & $1.9207 e-002$ & $1.6189 e-001$ \\
8 & $1.1985 e-004$ & $1.5547 e-003$ & $4.4413 e-004$ & $6.4158 e-003$ \\
12 & $6.4970 e-006$ & $6.6403 e-005$ & $3.2776 e-005$ & $4.0270 e-004$ \\
16 & $2.7904 e-007$ & $2.7133 e-006$ & $1.2055 e-006$ & $1.7195 e-005$ \\
20 & $9.2791 e-009$ & $8.8367 e-008$ & $5.5347 e-008$ & $9.3584 e-007$ \\
24 & $2.7288 e-010$ & $2.7397 e-009$ & $1.5502 e-009$ & $2.7927 e-008$ \\
\hline
\end{tabular}

The exact solution is given by

$$
u=\left\{\begin{array}{l}
r^{2}, \quad r \leq \frac{1}{2} \\
\left(1-\frac{1}{8 b}-\frac{1}{b}\right)+\frac{\left(r^{4} / 2+r^{2}\right)}{2}+\frac{C \log (2 r)}{b} \\
\quad r>\frac{1}{2} .
\end{array}\right.
$$

In this example, we have varying discontinuous coefficient and singular source term as well. Tables 4 and 5 show the numerical results for both Legendre and Chebyshev approximation for different values of $b$ and $C$. We observe that the errors in $L_{\omega}^{2}(\Omega)$ - and $H_{\omega}^{1}(\Omega)$-norm are decay exponentially regardless of discontinuous coefficients and singular source term. The exact solution, approximation, and its error for the Legendre case are plotted in Figures 5 and 6, for different values of $b$ and $C$.

Example 4 (circular interface 3 with jump condition [6]). Consider Laplace equation

$$
\begin{gathered}
u_{x x}+u_{y y}=0, \quad \text { in } \Omega, \\
u=0 \quad \text { on } \partial \Omega .
\end{gathered}
$$

The exact solution is given

$$
u(x, y)= \begin{cases}e^{x} \cos (y), & r \leq \frac{1}{2} \\ 0 . & r>\frac{1}{2}\end{cases}
$$

In this example we impose a jump in solution $u$ itself and also a jump in the normal derivative of $u$ as external constraints. Table 6 also shows the spectral convergence for the Legendre and Chebyshev pseudo-spectral element method although it has jump in solution and its normal derivative Table 7 . The exact solution, approximated one, and its error for the Legendre case of this example are plotted in Figure 7.

\section{Concluding Remarks}

In this paper, we proposed the pseudo-spectral collocation method for second order elliptic interface problems with 


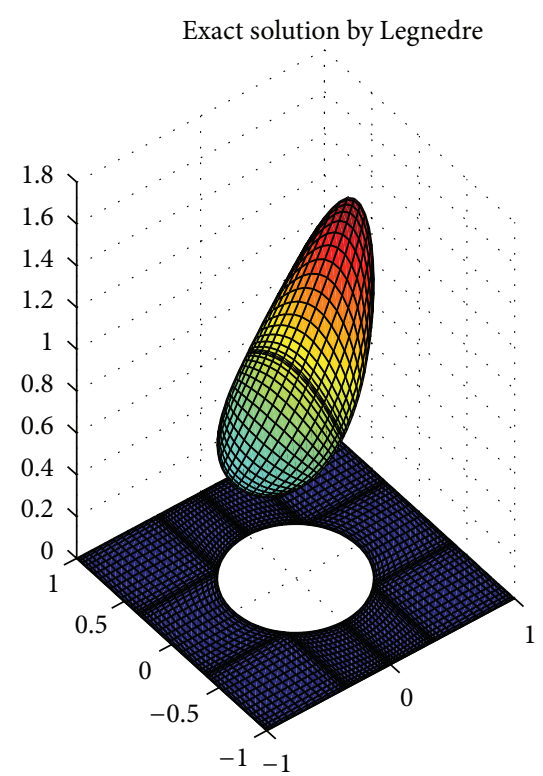

(a)

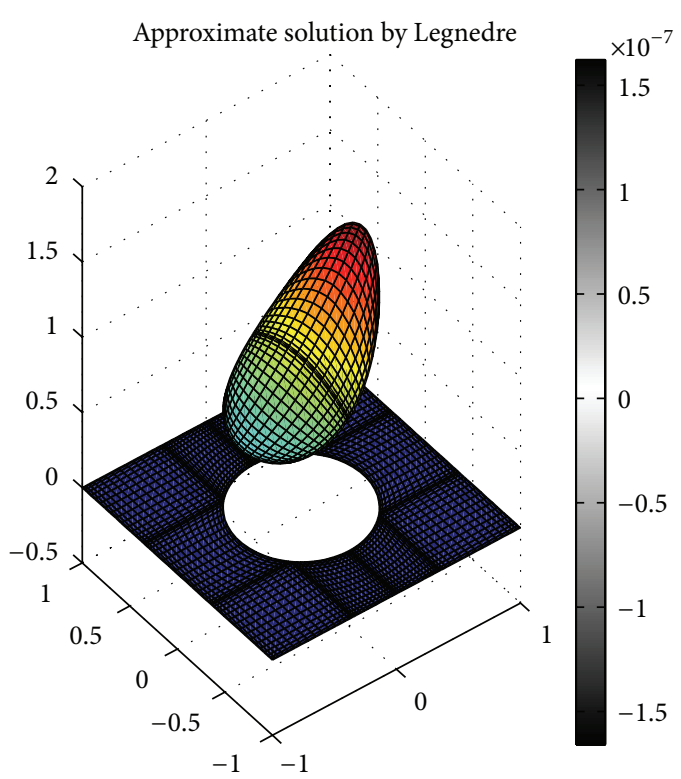

(b)

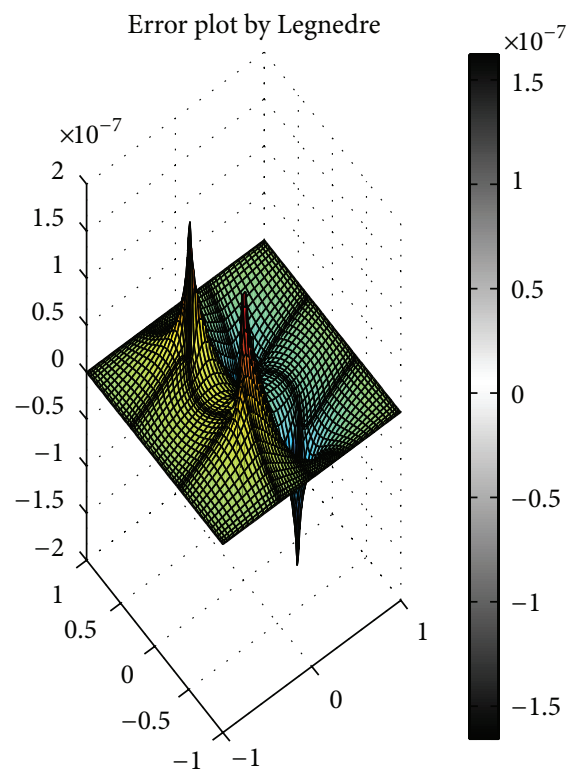

(c)

Figure 7: Exact solution, approximation solution, and its error for $N=18$.

discontinuous coefficients and singular source term. First we derived interface conditions in variable and its normal derivative and two equations defined on each subdomain decomposing the whole domain into two subdomains based on the interface. Then we obtain very simple algorithm applying the spectral collocation method to each equation. It is shown that the proposed method can easily applied to interface problem defined on curved domain by using Gordon and Hall transformation. This means that the method can be easily extended to spectral element collocation method to solve interface problems defined on a complicated domain. The numerical experiments also showed that the method has the spectral convergence with high accuracy. Furthermore, the method can be adopted to solve Stokes or Navier-Stokes equations with interface which can be studied in a coming paper.

\section{Conflict of Interests}

The authors declare that there is no conflict of interests regarding the publication of this paper.

\section{Acknowledgments}

The second author was supported by Kyungpook National University fund 2013 and the third author was supported by Basic Science Research Program through 
the National Research Foundation of Korea (NRF) funded by the Ministry of Education, Science and Technology (NRF2013R1A2009687).

\section{References}

[1] R. E. Ewing, "Problems arising in the modeling of processes for hydrocarbon recovery," in The Mathematics of Reservoir Simulation, pp. 3-34, SIAM, Philadelphia, Pa, USA, 1983.

[2] Z. Li, T. Lin, and X. Wu, "New Cartesian grid methods for interface problems using the finite element formulation," Numerische Mathematik, vol. 96, no. 1, pp. 61-98, 2003.

[3] B. F. Nielsen, "Finite element discretizations of elliptic problems in the presence of arbitrarily small ellipticity: an error analysis," SIAM Journal on Numerical Analysis, vol. 36, no. 2, pp. 368-392, 1999.

[4] D. W. Peaceman, Fundamentals of Numerical Reservoir Simulation, Elsevier, 1977.

[5] J. N. Reddy, Finite Element Method, McGraw-Hill, New York, NY, USA, 1993.

[6] R. J. LeVeque and Z. L. Li, "The immersed interface method for elliptic equations with discontinuous coefficients and singular sources," SIAM Journal on Numerical Analysis, vol. 31, no. 4, pp. 1019-1044, 1994.

[7] Z. Li, "The immersed interface method using a finite element formulation," Applied Numerical Mathematics, vol. 27, no. 3, pp. 253-267, 1998.

[8] B.-C. Shin and J.-H. Jung, "Spectral collocation and radial basis function methods for one-dimensional interface problems," Applied Numerical Mathematics, vol. 61, no. 8, pp. 911-928, 2011.

[9] A. Loubenets, T. Ali, and M. Hanke, "Highly accurate finite element method for one-dimensional elliptic interface problems," Applied Numerical Mathematics, vol. 59, no. 1, pp. 119-134, 2009.

[10] H. Huang and Z. Li, "Convergence analysis of the immersed interface method," IMA Journal of Numerical Analysis, vol. 19, no. 4, pp. 583-608, 1999.

[11] Z. Li and K. Ito, "Maximum principle preserving schemes for interface problems with discontinuous coefficients," SIAM Journal on Scientific Computing, vol. 23, no. 1, pp. 339-361, 2001.

[12] I. T. Angelova and L. G. Vulkov, "High-order finite difference schemes for elliptic problems with intersecting interfaces," Applied Mathematics and Computation, vol. 187, no. 2, pp. 824843, 2007.

[13] Z. Li, T. Lin, and X. Wu, "New cartesian grid methods for interface problems using the finite element formulation," Numerische Mathematik, vol. 96, no. 1, pp. 61-98, 2003.

[14] M. Hanke and A. Loubenets, "An immersed finite element method and its convergence for elliptic interface problems with discontinuous coefficients and singular sources," TRITA-CSC2007:3.

[15] D. Y. Kwak, K. T. Wee, and K. S. Chang, "An analysis of a broken $P_{1}$-nonconforming finite element method for interface problems," SIAM Journal on Numerical Analysis, vol. 48, no. 6 , pp. 2117-2134, 2010.

[16] M. Latige, T. Colin, and G. Gallice, "A second order Cartesian finite volume method for elliptic interface and embedded Dirichlet problems," Computers and Fluids, vol. 83, pp. 70-76, 2013.

[17] Z. G. Seftel, "A general theory of boundary value problems for elliptic systems with discontinuous coefficients," Ukrainian Mathematical Journal, vol. 18, no. 3, pp. 132-136, 1966.
[18] W. J. Gordon and C. A. Hall, "Transfinite element methods: blending-function interpolation over arbitrary curved element domains," Numerische Mathematik, vol. 21, no. 2, pp. 109-129, 1973.

[19] W. J. Gordon and C. A. Hall, "Construction of curvilinear co-ordinate systems and applications to mesh generation," International Journal for Numerical Methods in Engineering, vol. 7, no. 4, pp. 461-477, 1973. 


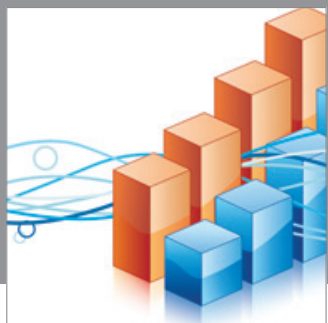

Advances in

Operations Research

mansans

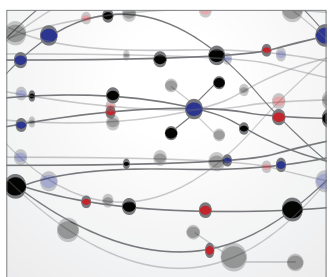

The Scientific World Journal
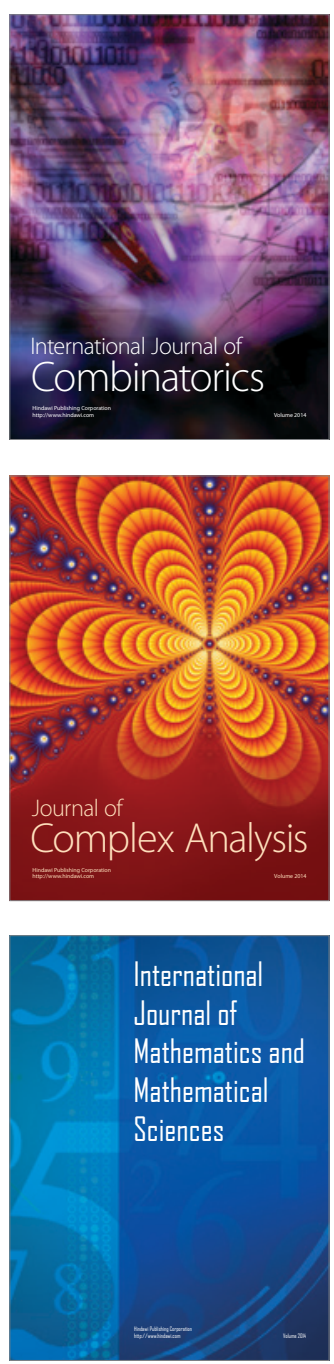
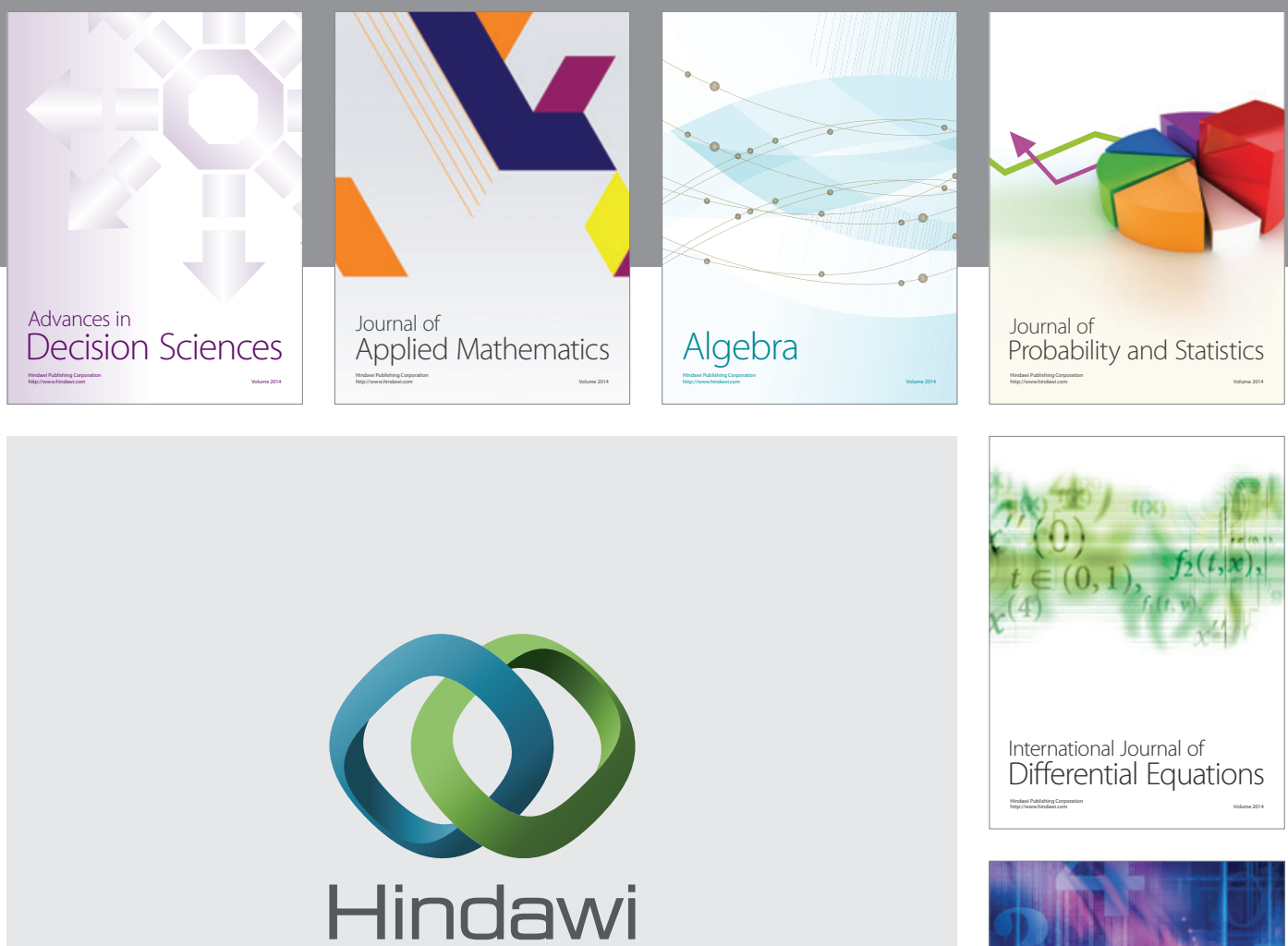

Submit your manuscripts at http://www.hindawi.com
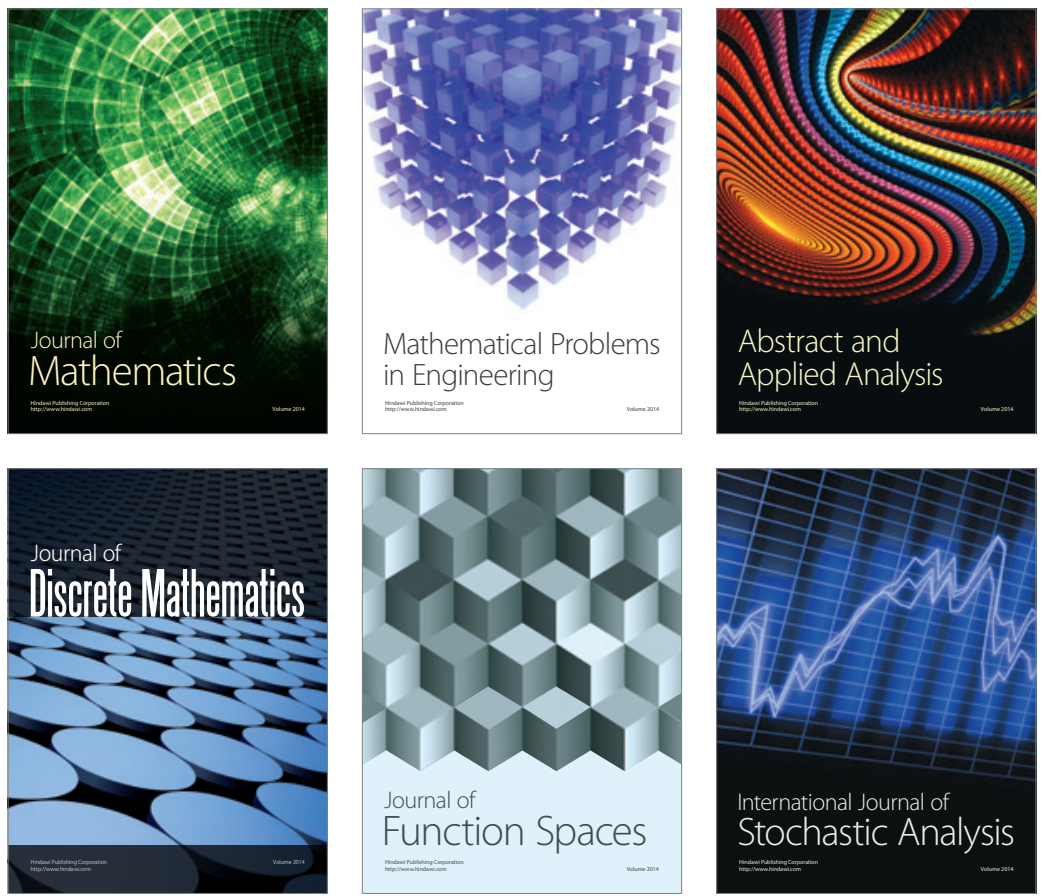

Journal of

Function Spaces

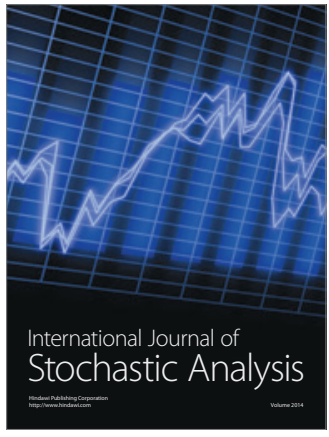

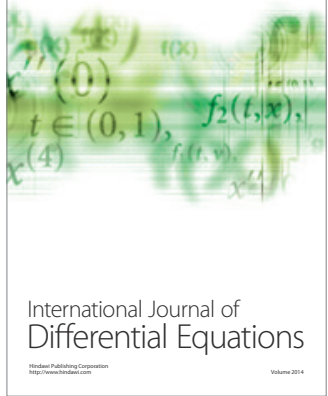
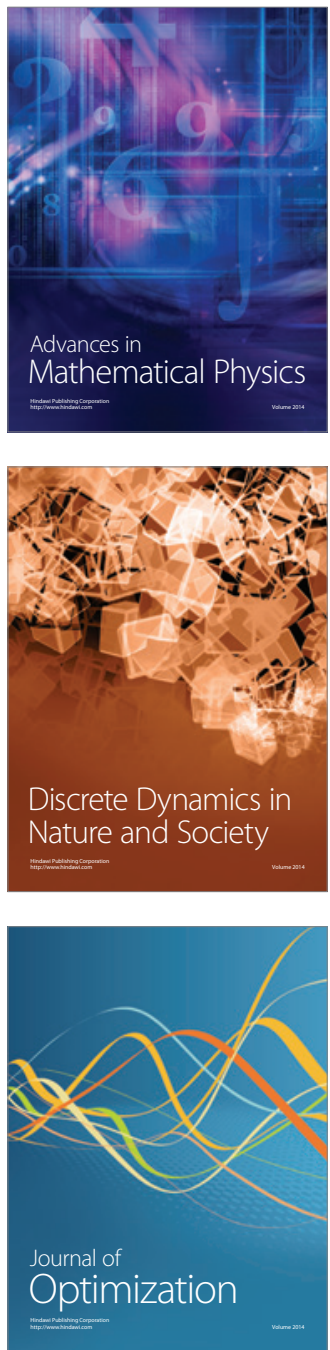